\title{
Incidence of retinopathy of prematurity (ROP) and risk factors associated to ROP in rural area
}

\author{
Punit Singh $^{1, *}$, Deepak Patel ${ }^{2}$ \\ ${ }^{1}$ Associate Professor, ${ }^{2}$ Professor, Dept. of Ophthalmology, SBKS Medical Institute \& Research Center, Vadodara, Gujarat, India
}

*Corresponding Author: Punit Singh

Email: punitsinghdr@yahoo.com

\begin{abstract}
Aim: Study of incidence, clinical staging and risk factors of ROP in rural area.

Materials and Methods: All premature new born infants at R.L.J. H Kolar were screened between July 2008 and June 2009 .The incidence, clinical staging and associated risk factors were studied. We compared our statistics with urban area statistics from literature.

Results: 210 children were screened. (43\%) of them were diagnosed as ROP amongst which (50\%) of them were having stage 1 ROP, $(33 \%)$ stage $2,(15 \%)$ of them were having APROP. $22.5 \%$ of them had risk factors among them PIH (40\%), anaemia $(30 \%)$ were common.

Conclusions: Incidence and risk factors are high in rural area compared to urban, so all premature infants should be screened thoroughly
\end{abstract}

Keywords: ROP, New born, Premature.

\section{Introduction}

Retinopathy of prematurity has been considered as one of the most common cause of blindness in children. Though in India has most advanced neonatal care but still babies with low birth weight and born prematurely are considering at higher risk of developing ROP. However, its incidence still remains low. ${ }^{1}$ The detailed information about the incidence rate of ROP and risk factors associated to it with clinical outcomes has been studied in CRYO-ROP trial. ${ }^{2}$ Lower birth weight and post - conceptional age are mainly responsible for manifestation and severity of ROP. It has been found in some of the research studies that almost $1 / 2$ of the babies with birth weight between $1.000 \mathrm{~kg}$ to $1.250 \mathrm{~kg}$ has developed ROP whereas this rate increases in babies with birth weight $<1.0 \mathrm{~kg}$, i.e. $81.6 \%{ }^{3}$

In utero, there is hypoxic state for the foetus. Vascular endothelial growth factor (VEGF) secreted in premature birth and due to this the retinal vessels grows. However if there is prolonged exposure of hypoxia to the immature retina of the infant the vessels do not develop and the retina remains in an avascular state. With such a prolonged exposure to hypoxia, the avascular retina becomes ischemic and further stimulates VEGF leading to abnormal arterial venous shunts and neovascularization.

Due to such advanced neonatal care unit the survival of premature babies has increased. Such babies are at a higher risk of developing ROP. If premature babies and low birth weight babies are not screened early it can lead to complete visual impairment. The main aim of this study is to know the incidence and associated risk factors of retinopathy of prematurity in rural area. It is also useful to increase the awareness and the need for its screening and treatment in rural area.

\section{Aims and Objectives}

1. To find the incidence rate of clinical staging of ROP.

2. To find the prevalence of risk factors associated to ROP in premature new born infant in rural area.

\section{Materials and Methods}

This study has been carried out at Neonatal care unit of RLJH and RC, Tamaka, Kolar, Sri Devaraj Urs Medical College for a period of six months.

\section{Inclusion Criteria}

1. All new-borns with birth weight $>2.0 \mathrm{~kg} \&<2.5$ $\mathrm{kg}$.

2. The birth of neonates is between the gestational age of 30-37 weeks.

3. Any new borns admitted with known risk factors for retinopathy of prematurity.

\section{Exclusion Criteria}

1. New born had any family history of exudative vitreoretinopathy.

2. New born had any family history of feature suggestive of Norries disease.

3. Congenital hydrocephalus.

4. Any parent of new born baby reject to give voluntary consent.

Before initiation of the study Ethical Committee Approval has been taken. In the present study we have screened total of 210 neonates for a period of six months, who were satisfying the all selection criteria of the study. Before enrolling the neonate in the study voluntarily written informed consent has been obtained from the parents and or guardian of the neonates.

Pupils were dilated using phenylephrine $2.5 \%$ and cyclopentolate $0.5 \%$ eye drops. All the new born were examined by the experienced ophthalmologist using indirect ophthalmoscopy and +20 D lens with scleral 
depressor after applying lid speculum and topical anaesthesia. Also screened by using RETCAM, a portable digital camera.

In this study classification of ROP was done by using international classification of retinopathy. Incidence, clinical staging and risk factors were recorded and analysed.

\section{Results}

In the present study total 210 children were screened who were satisfying all inclusion and exclusion criteria of the study and out of all enrolled children $90(45.71 \%)$ were diagnosed as ROP. It has been found in our study that almost $2 / 5^{\text {th }}$ children were affected with ROP. As shown in table 2, Stage 1 ROP was found in $46.87 \%$ neonates, $32.29 \%$ neonates had Stage 2 ROP, $14.58 \%$ had APROP, $4.16 \%$ having PLUS ROP and $2.08 \%$ having pre plus ROP respectively. Different maternal and neonatal risk factors have been found in 44 cases among the all 90 ROP cases. Pregnanacy induced hypertension was found to be a most commonest risk factor for ROP and it was found in $34.09 \%$ of cases. Similarly anemia was found in $31.81 \%$ whereas exchange of transfusion was found in $22.72 \%$ of cases and mechanical ventilation was found in $11.36 \%$ of cases.

Table 1: Incidence of ROP in present study

\begin{tabular}{|c|c|}
\hline Total no of cases screened & Total no. of ROP cases diagnosed \\
\hline 210 & $96(45.71 \%)$ \\
\hline
\end{tabular}

Table 2: Prevalence of different stages of ROP

\begin{tabular}{|c|c|c|c|c|c|}
\hline Staging & Stage 1 & Stage 2 & APROP & Pre plus & Plus \\
\hline No. of cases & $45(46.87 \%)$ & $31(32.29 \%)$ & $14(14.58 \%)$ & $2(2.08 \%)$ & $4(4.16 \%)$ \\
\hline
\end{tabular}

Table 3: Risk Factor associated with ROP

\begin{tabular}{|c|c|c|c|}
\hline $\begin{array}{c}\text { Pregnancy induced } \\
\text { hypertension }\end{array}$ & Anaemia & Exchange transfusion & Mechanical ventilation \\
\hline $15(34.09 \%)$ & $14(31.81 \%)$ & $10(22.72 \%)$ & $5(11.36 \%)$ \\
\hline
\end{tabular}

\section{Discussion}

One of the major causes of blindness in children is Retinopathy of Prematurity (ROP).

Lower birth weight, premature babies and prolonged Ventilation has strong association with ROP. Studies has been suggested that neonates had birth weight $<1.0 \mathrm{~kg}$ and with gestational age of less than 30 week are considered at higher risk of developing ROP. ${ }^{8-}$ ${ }^{10}$ Some studies also suggesting that lack of knowledge and awareness about pre natal and ante natal care also leads to the risk of developing ROP and also increase the rate of visual impairments due to ROP. ${ }^{8,9}$ With improving neonatal care units there is increase in rate of survival of preterm and low birth weight babies. ${ }^{10-14}$

In utero, there is hypoxic state for the fetus. When premature birth occurs, vascular endothelial growth factor (VEGF) is secreted which in turn helps the growth of the retinal vessels. ${ }^{14-16}$ However if there is prolonged exposure of hypoxia to the immature retina of the infant the vessels do not develop and the retina remains in an avascular state. ${ }^{17}$ With such a prolonged exposure to hypoxia, the avascular retina becomes ischemic and further stimutaes VEGF leading to abnormal arterial venous shunts and neovascularisation. ${ }^{18-20}$

Compromised fetal growth and nutrition due to pregnancy induced hypertension and toxemia causes placental infarcts and compromises fetal blood flow. This in turn leads to intra-uterine growth retardation (IUGR). About 50\% of low birth weight babies have chances to develop ROP. About $90 \%$ of infants weighing less than 750 grams develop retinopathy of prematurity. ${ }^{21,22}$

Duration of oxygen given to neonates also increased the risk of ROP. The VGEF produces by retinal vessel induces neo-vascularization at the border between vascularized and non-vascularized retina which may eventually lead to retinal detachment. ${ }^{9,15,18,23}$ Anemia is an independent factor for the development of ROP. Anemia induces hypoxia in the retinal tissue and hence predispose to ROP.

There is a controversial role of blood transfusion in development and progression of retinopathy of prematurity. The premature babies may need more live support and blood transfusion than the normal babies. ${ }^{15}$ These babies who are exposed to adult blood may develop severe retinopathy than the normal infants. The incidence of retinopathy may also be more in babies who have received blood transfusion, However, theoretical risks associated with factors such as volume and iron load may place infants who receive more transfusions at higher risk for retinopathy of prematurity. ${ }^{13,20}$

Adult blood when given to infants may cause oxygen dislocation leading to ROP. Other risk factors include apnea, sepsis, patent ductus arteriousus, congenital heart disease, intra uterine growth retardation, respiratory distress syndrome and blood transfusions. $^{7}$ 
Recent studies by Lofqvist et al have shown that infants whose postnatal gain weight is less than expected are at increased risk.

Gestational age <32 wks, pregnancy induced hypertension, oxygen administration, anemia and blood transfusion were found to have major and significant role in ROP.

In our study we found that the incidence rate of ROP was significantly higher in neonates with the above maternal and neonatal risk factors. Screening of such neonates with appropriate methods can be considered as a cost effective and thus reduced the incidence of ROP and helped to treat the disease. It has been recommended that first screening should be done as early as 3-4 weeks of postnatal age or 34-35 weeks post conceptional age. Also continuous follow up needed to treat ROP and thus avoiding visual impairment from it.

\section{Conclusion}

Prevention of premature birth, prevent pregnancy induced hypertension, appropriate use of ventilation and oxygen therapy may reduce risk and severity of ROP. It is very essential for each ophthalmologist to analysis each risk factor associated to ROP to predict the course and development of the disease.

\section{References}

1. Charan R, Dogra MR, Gupta A, Narang A. The incidence of retinopathy of prematurity in a neonatal care unit. Indian journal of Ophthalmol. 1995;43:123-126.

2. Palmer EA, Flynn JT, Hardy RJ. Incidence and early courses of retinopathy of prematurity. Ophthalmology. 1991;98:1628-40

3. Rekha SS, Battu RR. Retinopathy of prematurity: Incidence and risk factors. Indian Pediatr. 1996;33:9991003.

4. Alme AM, Mulhern ML, Hejkal TW. Outcome of retinopathy of prematurity patients following adoption of revised indications for treatment. BMC Ophthalmology. 2008;8:23.

5. Schaffer DB, Palmer EA, Plolsky DF. Prognostic factors in the natural course of retinopathy of prematurity. Ophthalmology.1993;100:230-7.

6. Shah VA, Yeo CL, Ling YL. Incidence of risk factors of retinopathy of prematurity among very low birth weight infants in Singapore. Ann Accd Med Singapore. 2005;34:169-78.

7. Chaudhari S, Patwardha V, Vaidya U, Kadam S, Kamat A. Retinopathy of prematurity in a Tertiary care center. Indian Pediatr. 2009;46:219-223.

8. Lundgren P, Stoltz Sjostrom E, Domellof M. WINROP identifies severe retinopathy of prematurity at an early stage in a nation-based cohort of extremely preterm infants. PLoS One. 2013;8(9):e73256.

9. Anuk Ince D, Gülcan H, Hanta D, Ecevit A, Akkoyun I, Kurt A. Poor postnatal weight gain predicts stage 3+ retinopathy of prematurity in very low birth weight infants. The Turkish Journal of Paediatrics. 2013;55(3):304-308.

10. Dhaliwal CA, Fleck BW, Wright E, Graham C, McIntosh $\mathrm{N}$. Retinopathy of prematurity in small-for-gestational age infants compared with those of appropriate size for gestational age. Arch Dis Child Fetal Neonatal Ed. 2009;94(3):193-195.

11. Hussain N, Clive J, Bhandari V. Current incidence of retinopathy of prematurity. Pediatric. 1999;104(3):e26.

12. STOP-ROP. Supplemental Therapeutic Oxygen for Prethreshold Retinopathy of Prematurity, a randomized, controlled trial. I: primary outcomes. Pediatrics. 2000;105(2):295-310.

13. Kim TI, Sohn J, Pi SY, Yoon YH. Postnatal risk factors of retinopathy of prematurity"; Paediatr Perinat Epidemiol. 2004;18(2):130-134.

14. Shah VA, Yeo CL, Ling YL, Ho LY. Incidence, risk factors of retinopathy of prematurity among very low birth weight infants in Singapore. Ann Acad Med Singapore. 2005;34(2);169-178.

15. Gilbert C, Fielder A, Gordillo L. Characteristics of infants with severe retinopathy of prematurity in countries with low, moderate, and high levels of development: implications for screening programs. Pediatrics. 2005;115(5):518-525.

16. Repka MX, Summers CG, Palmer EA. The incidence of ophthalmologic interventions in children with birth weights less than 1251 grams. Results through 5 1/2 years. Cryotherapy for Retinopathy of Prematurity Cooperative Group. Ophthalmology. 1998;105(9):16211627.

17. Cryotherapy for Retinopathy of Prematurity Cooperative Group. Multicenter Trial of Cryotherapy for Retinopathy of Prematurity: ophthalmological outcomes at 10 years. Arch Ophthalmol. 2001;119(8):1110-1118.

18. Tadesse M, Dhanireddy R, Mittal M, Higgins RD. Race, Candida sepsis, and retinopathy of prematurity. Biol Neonate. 2002;81(2):86-90.

19. Bizzarro MJ, Hussain N, Jonsson B. Genetic susceptibility to retinopathy of prematurity. Pediatrics. 2006;118(5);1858-1863.

20. Hellstrom A, Hard AL, Engstrom E. Early weight gain predicts retinopathy in preterm infants: new, simple, efficient approach to screening. Pediatrics. 2009;123(4):638-645.

21. International Committee for the Classification of Retinopathy of Prematurity. The International Classification of Retinopathy of Prematurity revisited. Arch Ophthalmol. 2005;123(7):991-999.

22. AAP, AAO, and AAPOS. Screening examination of premature infants for retinopathy of prematurity. Pediatrics. 2006;117(2):572-6.

23. Subhani M, Combs A, Weber P. Screening guidelines for retinopathy of prematurity: the need for revision in extremely low birth weight infants. Pediatrics; 2006;107(4);656-659.

How to cite this article: Singh $P$, Patel D. Incidence of retinopathy of prematurity (ROP) and risk factors associated to ROP in rural area. Indian J Clin Exp Ophthalmol. 2018;4(4):551553. 